\title{
1 Flexible Modulation of Neural Variance Facilitates Neuroprosthetic Skill
}

2 Learning

Albert K. You ${ }^{1}$, Bing Liu ${ }^{2}$, Abhimanyu Singhal ${ }^{3,4}$, Suraj Gowda ${ }^{4}$, Helene Moorman ${ }^{5}$, Amy Orsborn $^{6,7,8}$, Karunesh Ganguly ${ }^{9}$, Jose M. Carmena ${ }^{1,4,5, *}$

${ }^{1}$ UC Berkeley-UCSF Joint Graduate Program in Bioengineering, University of California, 8 Berkeley, Berkeley, CA 94720, USA

$9 \quad{ }^{2}$ Department of Neurobiology, Duke University, Durham, NC 27710, USA

${ }^{3}$ Department of Physics, University of California, Berkeley, Berkeley, CA 94720, USA

${ }^{4}$ Department of Electrical Engineering and Computer Sciences, University of California, Berkeley, Berkeley, CA 94720, USA

${ }^{5}$ Helen Wills Neuroscience Institute, University of California, Berkeley, Berkeley, CA 94720, USA

${ }^{6}$ Department of Electrical and Computer Engineering, University of Washington, Seattle, WA 98195, USA

${ }^{7}$ Department of Bioengineering, University of Washington, Seattle, WA 98195, USA

${ }^{8}$ Washington National Primate Research Center, Seattle, WA 98121, USA

${ }^{9}$ Weill Institute for Neurosciences, University of California, San Francisco, San Francisco, CA 94143, USA

*Correspondence: jcarmena@ berkeley.edu (J.M.C.)

\section{SUMMARY}

24 One hallmark of natural motor control is the brain's ability to adapt to perturbations ranging

25 from temporary visual-motor rotations to paresis caused by stroke. These adaptations require

modifications of learned neural patterns that can span the time-course of minutes to months.

Previous work with brain-machine interfaces (BMI) has shown that over learning, neurons

28 consolidate firing activity onto low-dimensional neural subspaces, and additional studies have 
33 allowed us to track how specific populations of neurons changed firing patterns as task

34 performance improved. In each experiment, neural activity was transformed into cursor

35 kinematics using decoding algorithms that were periodically readapted based on natural arm

36 movements or visual feedback. We found that decoder changes caused neurons to increase

37 exploratory-like patterns on within-day timescales without hindering previously consolidated

38 patterns regardless of task performance. The flexible modulation of these exploratory patterns in

39 contrast to relatively stable consolidated activity suggests a simultaneous exploration-

40 exploitation strategy that adapts existing neural patterns during learning. 


\section{INTRODUCTION}

42 Our brains have an intrinsic ability to adapt to perturbations. However, the degree to which we can adapt depends on the similarity to previously learned behaviors and the time allowed for adaptation (Izawa et al., 2008; Kording et al., 2007; Shadmehr et al., 2010; Wei and Körding, 2009). Brain-machine interfaces (BMI) allow us to interrogate specific populations of neurons and examine how they adapt during learning (Athalye et al., 2020; Golub et al., 2016; Shenoy and Carmena, 2014). BMI decoders translate neural activity ranging from firing rates to field potentials into actions taken by an end effector (e.g. computer cursor or robotic arm). It has been shown in multiple studies that the brain adapts and forms stereotyped firing patterns over learning (Carmena et al., 2003; Chase et al., 2012; Ganguly and Carmena, 2009; Jarosiewicz et al., 2008; Koralek et al., 2012; Orsborn et al., 2014; Taylor, 2002; Wander et al., 2013). We refer to these stereotyped patterns as neuroprosthetic maps (Ganguly and Carmena, 2009; Ganguly et

$$
\text { al., 2011; Orsborn et al., 2014). More recent work has shown how the brain coordinates the }
$$
activity of neurons over learning and collapse upon a low-dimensional neural subspace as performance improves when given a fixed decoder (Athalye et al., 2017; Oby et al., 2019).

In addition, similar results regarding neuroprosthetic map formation have been shown in BMI

58 experiments where decoders are periodically readapted to fit changes in the neural population

59 (Orsborn et al., 2014). We refer to these paradigms as "2-learner systems." In these experiments,

60 closed-loop decoder adaptation (CLDA) was used to refit decoder weights to updated neural

61 intentions at the start of the day, allowing for quick improvements in BMI performance (Dangi et

62 al., 2013; Gilja et al., 2012; Moorman et al., 2017; Orsborn et al., 2012, 2014). However, while

63 CLDA incrementally fits the overall control space closer to the neural subspace, the decoder 
64 weights of individual neurons could be affected due to changes in the ensemble of neurons, the

65 variance of neural activity, or sources of noise. Thus, new decoder weights inherently introduce

66 slight perturbations to individual neurons even if the overall population activity is better

67 modeled. In these experiments, task performance continued to improve alongside these decoder

68 refits. Thus, we asked how neurons adapt given slight decoder changes throughout learning.

69 Furthermore, what is the timescale of this adaptation? Refitting a new decoder happens on the

70 timescale of minutes. Thereafter, the decoder is fixed for the duration of the day and sometimes

71 held fixed for multiple days. How do neurons respond to relatively fast changes in decoder

72 weights and still maintain the ability to consolidate firing patterns over longer multi-day

73 timescales?

74

75 Since CLDA fits to the neural intentions of the subject, one can assume that any changes in

76 weights in the decoder are mainly rotations within the low-dimensional neural subspace,

77 sometimes referred to as the intrinsic manifold. As reported in recent studies, such perturbations

78 are quickly learned within-day whereas out-of-manifold perturbations are more difficult for

79 neurons to adapt indicating resistance to changes in the neural subspace (Golub et al., 2018; Oby

80 et al., 2019; Sadtler et al., 2014). Thus, neurons may be adapting to periodically refit decoders as

81 they would to fixed decoders since the control spaces are not significantly perturbed by new

82 decoder weights. However, these decoder weight changes introduce errors on individual neurons

83 such as misalignment of preferred tuning directions, potentially creating slight out-of-manifold

84 perturbations. In order to adapt to these errors, we hypothesize that neurons increase their neural

85 firing rate variance while maintaining relatively stable, coordinated population-level firing

86 patterns on short, within-day timescales. 
88 To observe the effects of perturbations on the consolidation of neural firing patterns throughout

89 learning, we analyzed data from three BMI experiments performed on a total of five male rhesus

90 macaques. These experiments refit decoders at various frequencies, ranging from repeated

91 decoder swaps within-day to performing CLDA at the beginning of each day (Figure 1). These

92 differences allowed us to gain increased insight on how neurons adapt on short versus long-term

93 timescales. As expected, our results show that neurons increased neural variance immediately

94 after decoder-refitting. However, the variance dropped as the decoder was held fixed, thereby

95 increasing the fraction of neural variance that was aligned with the low-dimensional manifold.

96 Importantly, these manifolds did not change within day nor across many days. Rather, neural

97 variance was slowly collapsed onto a relatively stable neural subspace over learning. These

98 flexible changes in neural variance alongside stable low-dimensional neural spaces from the

99 onset of learning suggest a concomitant exploration and exploitation strategy during motor

100 learning, where new neural patterns are formed by adapting preexisting patterns. 


\section{RESULTS}

104

105

106

107

108

109

110

111

112

113

114

115

116

117

In this study we analyzed data from three previous studies (Ganguly and Carmena, 2009;

Orsborn et al., 2014; Moorman and Gowda et al., 2017) involving five monkey subjects. In each experiment, decoders were periodically changed and monkeys were trained over the time-course of days thus allowing us to ask how neural consolidation occurs alongside changes in decoder weights over learning. Furthermore, all three experiments consisted of center-out tasks in 2D space whereby monkeys were instructed to move the effectors to eight peripheral targets (Figure 1B). In Experiment 1 (Figure 1C, green), two biomimetic decoders were used each day. The animal (Monkey P) had previous experience with one decoder (DecoderoLD) but was naïve to the second (Decoder NEw). While performance with the old decoder was stable and high across all four days, control with the new decoder improved across days (Figure 1C, green).

In Experiment 2 (Figure 1C, blue), both monkeys ( $\mathrm{J}$ and $\mathrm{S}$ ) learned to control the BMI with suboptimal decoders as indicated by their initial performance on Day 1 (Figure 2B). Closed-loop decoder adaptation (CLDA) was used to intermittently refit decoder weights over the time-course of learning (Figure 1C, middle, red dots). Note that while Monkey $\mathrm{J}$ had two time series of data collected, the $40 \%$ CLDA condition (indicating $40 \%$ performance accuracy after CLDA training on the first day) occurred the day after the $20 \%$ CLDA condition was completed.

Finally, in Experiment 3 (Figure 1C, purple), two monkeys ( $\mathrm{C}$ and $\mathrm{G}$ ) moved a kinematically redundant 4 degree-of freedom (DOF) virtual arm to the same set of targets as in the previous experiments. CLDA was performed for roughly 10 minutes at the beginning of each day leading to high performance from the first day. However, decoders weights were still changed from day 
to day; correlations of these weights are shown in Supplemental Figures 4,5. While the percent correct was saturated from the first day ( $\sim 80 \%$ for both animals), their reach times continued to decrease across training indicating skill improvement (Figure 1C, purple).

\section{Factor Analysis Predicts Flexible Modulation of Consolidated Patterns}

As it has been previously reported (Athalye et al., 2017; Golub et al., 2018; Oby et al., 2019; Sadtler et al., 2014), factor analysis (FA) provides an intuitive model for how neurons coordinate firing patterns. In short, FA analyzes population-level firing trends and decomposes each neuron's firing activity into covarying (shared) and uncorrelated (private) components (Figure 2A). Importantly, since we were interested in how neural populations adapted over learning, only units that were stably recorded across the entire timeseries for each experiment were used. Geometrically, this shared space (or often referred to as an intrinsic manifold) refers to a lowdimensional neural subspace where neurons fire in correlated ways (Figure 2B). Subsequently, the private variance (PV) captures the variance of firing rates of a neuron that is independent from other neurons and has been suggested to be a correlate of exploratory patterns (Athalye et al., 2017).

Previous work in the field has focused primarily on the effects of BMI learning on shared spaces and it has been shown that decoders that align with the neural shared space (i.e. intrinsic manifold) can be learned more readily within a day whereas decoders that are explicitly out of alignment with the intrinsic manifold cannot be as quickly learned (Sadtler et al., 2014). Since our experiments rely on biomimetic movements and CLDA to fit effector kinematics to the neural intentions of the animal, we would expect the decoders used in these experiments to be 
149 roughly within the intrinsic manifold and therefore we would expect stable alignment of the

150 shared space (Figure 2B). Hypothetically, if a decoder was perfectly fit to the neural intentions of

151 an animal, we would expect no changes in either the angle or the size of the shared space.

152 However, due to the high dimensionality of the neural populations recorded during these BMI

153 tasks, decoder weights change slightly upon a decoder refit. Thus, we hypothesize that since the

154 intrinsic manifold is relatively stable on short timescales, neurons must increase their overall

155 firing rate variance (including both PV and shared variance) to accommodate perturbations in the

156 control space (i.e. decoder weights) (Figure 2B, middle). Subsequently, decoders that are less

157 aligned with the intrinsic manifold would cause a larger increase in overall neural variance.

Additionally, work by Athalye et al., 2017 showed that neurons increased their proportion of shared variance to total variance (SOT) over learning with a fixed decoder, indicating an emergence of coordinated or consolidated neural activity. While CLDA is used to periodically adapt the decoder weights, animals are able to learn the task over the time course of days (Orsborn et al., 2014). Together, this leads to the hypothesis that SOT increases even in the context of a 2-learner system (Figure 2B, right). Furthermore, since task performance is comparable before and after CLDA, some neural adaptation (e.g. changes in total variance) must

166 occur within the same day (Figure 2B, middle). In summary, we expect the neural variance of 167 BMI input neurons to increase as a function of decoder unfamiliarity on a short timescale while 168 shared structures are preserved and consolidated over a longer timescale. 
171 Past work has suggested that PV is linked with exploratory neural patterns that are less goal-

172 potent than shared activity but sufficient for achieving target hits (Athalye et al., 2017). Since

173 decoder refitting inherently causes some small perturbations in the decoder readout, we would

174 expect exploratory patterns to be engaged to accommodate increases in error. Congruent with

175 past results, we found that the PV increased each time the monkeys controlled the BMI with a

176 new decoder (Figures 3A,C,E). In Experiment 1, training with Decoder ${ }_{\mathrm{NEW}}$ led to higher PV in

177 each of the four days compared to training with Decoder ${ }_{O L D}$, which was already well-learned (t-

178 test, $\mathrm{p}=1.3 \mathrm{e}-7)$. In Experiment 2, the PV was calculated each day the decoder was refit and all

179 days following the perturbation (Figure 3C). The PV consistently increased the day following a

180 decoder refit for both monkeys, though only Monkey $\mathrm{J}$ had enough perturbations to test for

181 significance (t-test; Monkey $\mathrm{J}, \mathrm{p}=0.0334)$. Lastly, in Experiment 3, where a new decoder was

182 refit each day, the magnitude of PV change was correlated with the amount of change in decoder

183 weights (Figure 3E). Decoders that were similar to ones previously learned resulted in smaller

184 PV changes (correlation; Monkey $\mathrm{C}, \mathrm{R}=-0.70, \mathrm{p}=2 \mathrm{e}-4$; Monkey $\mathrm{G}, \mathrm{R}=-0.63, \mathrm{p}=6.3 \mathrm{e}-3$ ).

185 Implicit in this result is that PV decreases when more familiar (or fixed) decoders are used. We

186 found that indeed PV decreased when the task switched to using decoders that were more

187 familiar (Supplemental Figure 1A) or fixed for a period of days (Supplemental Figure 1B)

188 echoing results from Athalye et al., 2017.

190 Remarkably, these changes in PV were exhibited on the timescale of minutes. Private variance

191 decreased within the same day when an unfamiliar decoder was replaced with a well-learned

192 decoder (Supplemental Figure 1A). Conversely, switching back to the unfamiliar decoder the 
193 following day increased the PV (Figure 3A). These results show the flexibility of neural

194 variance, which may be required when adapting to new tasks or decoders.

196 Neural Exploration Adapts Consolidated Patterns

197 We asked how neural consolidation emerged in a 2-learner system despite frequent changes in 198 neural firing patterns due to changes in decoder weights. To track the proportion of neural 199 activity that fired in coordinated patterns, we normalized the shared variance by the total neural 200 variance (SOT). Thus, increases in SOT act as a measure for how covaried neurons' firing 201 activities are and can be thought of as the degree of consolidation of neural patterns (Athalye et 202 al., 2017). We found that while PV was modulated due to decoder changes, the SOT remained 203 stable on within-day timescales in Experiment 1 where decoders were switched in the middle of 204 the day (Figure 3B). Together, these results indicate a scaling of the total variance (including 205 shared variance) when a perturbation arises as predicted in Figure 2B (middle). In addition, SOT 206 increased in experiments where a single decoder was used each day (Figures 3D, F) (regression, 207 Monkey J, $\mathrm{R}^{2}=0.46, \mathrm{p}=2.8 \mathrm{e}-4$; Monkey $\mathrm{S}, \mathrm{R}^{2}=0.81, \mathrm{p}=6.1 \mathrm{e}-3$; Monkey $\mathrm{C}, \mathrm{R}^{2}=0.435, \mathrm{p}=$ $2083 \mathrm{e}-4$; Monkey $\left.\mathrm{G}, \mathrm{R}^{2}=0.829, \mathrm{p}=3.9 \mathrm{e}-7\right)$. The decoupling of changes in PV and SOT over different timescales suggests that neural exploration and exploitation can simultaneously occur,

210 and increased exploration does not occur at the expense of consolidated patterns. In contrast,

211 increases in SOT over the time-course of many days despite flexible changes in PV may suggest

212 that exploratory patterns consolidate over time and that neurons are adapting learned behaviors

213 rather than generating completely novel patterns. 
215 Furthermore, we asked if the shared spaces were stable across different decoders or if poor

216 performance with a new decoder would lead to adaptation to a different low-dimensional space.

217 To answer this question, we computed the "shared alignment" between the neural subspaces used

218 to control the two decoders in Experiment 1 (Supplemental Figure 2A). We found that the shared

219 spaces used to control the task were nearly identical despite large differences in task performance

220 (Supplemental Figure 2B). Similarly, we compared the shared alignment between adjacent days

221 and across days for Experiments 2 and 3 and found that shared spaces were stable on short and

222 long timescales (Supplemental Figure 2B-D). In all cases, the shared alignment was high in

223 adjacent blocks or days ( 0.8 across all animals), suggesting that neurons rely heavily on

224 preexisting patterns regardless of task performance.

225

226

Modulation Depth Facilitates Changes in Neural Variance

227 Finally, we asked how neurons are able to flexibly modulate their neural variance on short,

228 within-day timescales. Intuitively, our results imply that the dynamic range of firing rates across

229 all neurons must also be flexible to account for changes in neural variance. That is, solely

230 increasing the mean firing rate of neurons does not necessarily increase the variance. Rather, we

231 would expect some modulation of firing rates. Past literature has shown preferred direction

232 tuning in BMI neurons. The dynamic range of a neuron's tuning curve is often referred to as the

233 modulation depth (MD) of the neuron (Carmena et al., 2003; Ganguly and Carmena, 2009;

234 Ganguly et al., 2011; Orsborn et al., 2014). Past work has shown MD to change over many days

235 of BMI learning. However, given the flexibility of the neural variance, we hypothesized that MD

236 could also change on a shorter timescale. 
238 We calculated the preferred tuning models of each neuron by fitting a cosine tuning curve to the

239 mean firing rates corresponding to cursor velocity directions (Ganguly and Carmena, 2009;

240 Ganguly et al., 2011; Georgopoulos et al., 1988; Orsborn et al., 2014; Serruya et al., 2002). The

241 MD of each neuron was then determined by taking the peak-to-peak amplitude of the cosine

242 model. As predicted, we found that the MD was tightly coupled to the PV (Figure 4A)

243 (correlation; Monkey $\mathrm{C}, \mathrm{R}=0.86, \mathrm{p}<1 \mathrm{e}-5$; Monkey $\mathrm{G}, \mathrm{R}=0.63, \mathrm{p}=7 \mathrm{e}-3$ ). Furthermore, the

244 changes in MD were coupled to the changes in PV and was also able to change on a within-day

245 timescale (Figures 4B, C) (correlation; Monkey J, R =0.63, p = 0.0013; Monkey C, R =0.73, p

$246=1 \mathrm{e}-4)$. Notably, regression lines very closely intersected the origin indicating that MD does not

247 inherently increase due to task learning but could be due to modulation of PV. These results

248 suggest that neurons are able to quickly increase the firing rates specifically in their preferred

249 directions in response to perturbations in the control of the BMI. This increase in MD can be

250 thought of as a "stretching" of the tuning curve or an amplitude gain that increases the variance

251 of firing rate in the preferred direction without necessarily affecting the overall mean firing rate

252 of the neuron.

253

254 


\section{DISCUSSION}

256 Previous work has shown BMI performance to improve over days alongside decoder re-

257 adaptations (Oby et al., 2019; Orsborn et al., 2014). Here, we asked how neurons changed firing

258 patterns over learning to allow for these performance improvements despite changing decoder

259 weights. Our results support our hypothesis that motor cortex flexibly modulates neural variance

260 when errors arise (due to decoder re-adaptation) without eroding preexisting coordinated patterns

261 useful for generating goal-potent actions. Thus, at least in a 2-learner context, BMI learning

262 involves leveraging exploratory patterns to adapt learned neural patterns rather than generating

263 new ones.

265 Across all three experiments and five monkeys, the shared over total variance ratio (SOT) was

266 remarkably stable across learning while private variance (PV) reliably fluctuated whenever a

267 different decoder was introduced (Figure 3). Moreover, changes in PV were dependent on how

268 different the decoders were from previously learned control spaces (Figure 3E). This was

269 particularly noticeable in Experiment 1 where PV drastically changed within the same day while

270 SOT remained unchanged within and across days. Notably, SOT did increase over longer

271 timescales (i.e. Experiments 2 and 3) where perturbations were smaller. Experiment 1 used two

272 separate decoders, with a new one introduced after achieving expert control with the first

273 decoder. Furthermore, decoder perturbations happened each of the four days, each day ending

274 with the previously learned decoder. The frequency and magnitude of these perturbations may

275 have hindered consolidation effects observed in the other two experiments. Nonetheless, the SOT

276 was stable within and across days and did not decrease due to repeated perturbations. 
Also as predicted, we observed no changes in the low-dimensional neural subspace both withinday and across days (Supplemental Figure 2B-D). To quantify any changes, we calculated the "shared alignment" which represented the magnitude of the projection of one manifold onto another. In all three experiments, the alignment between any two adjacent blocks (or days for Experiments 2 and 3) were roughly 0.8 0.9 indicating similar subspaces on short timescales. These alignments were also similar across days suggesting minimal rotation of the manifold even over the time-course of learning.

Our results also suggest that modulation depth (MD) may play a critical role in facilitating fast changes in neural variance. While past studies have repeatedly shown changes in MD over learning, it has been unclear what the exact role of this may be. We found that the MD was tightly correlated with PV. More specifically, changes in PV were closely tracked by directly

290 proportional changes in MD (Figure 4). Importantly, fluctuations in both were fast (compared to 291 neural consolidation rates) in both increasing and decreasing directions (Figures 2, 4,

292 Supplemental Figure 1). Intuitively, increasing MD increases the dynamic range of neural firing 293 rates, allowing for higher total neural variance. In contrast to past studies showing overall 294 changes in MD over learning (Ganguly and Carmena, 2009; Ganguly et al., 2011; Orsborn et al., 295 2014), these fast changes in modulation depth indicate that the ability to fluctuate firing rates may be an inherent property of motor cortex rather than a learned behavior. neuroprosthetic learning and motor adaptation may have similar underlying mechanisms. 
Studies have shown the importance of subcortical structures and the cerebellum in adaption to motor perturbations and BMI tasks (Koralek et al., 2012; Nowak et al., 2007; Shadmehr and Krakauer, 2008). Futhermore, past studies provide evidence on the role of the basal ganglia for refining cortical patterns during BMI on shorter and longer timescales (Athalye et al., 2018, 2020; Costa et al., 2004; Ölveczky et al., 2011). In a motor adaptation task then, it is possible these structures are turning the "variability knob" in motor cortex. If we consider the production of motor actions as a combination of consolidated activity from the motor system with some exploratory variability (Fee and Goldberg, 2011; Neuringer et al., 2000), then our results suggest that PV may be a neural correlate of the latter. Furthermore, changes in neural variance might reflect an increased gain in exploratory variability without directly affecting learned motor behaviors. In such a scenario, neuroprosthetic learning - particularly with closed-loop decoder adaptation (CLDA) - may be encouraging changes from subcortical structures we see manifested as changes in motor cortex.

Our results are complementary with those from previous studies (Athalye et al., 2017; Golub et al., 2018; Oby et al., 2019; Sadtler et al., 2014). It has been shown that intrinsic manifolds are relatively stable and thus are less able to adapt to decoders that are explicitly out of that manifold (Sadtler et al., 2014). While learning out-of-manifold decoders is difficult within-day, it has also been shown that these shared spaces can rotate over many days of training (Athalye et al., 2017; Oby et al., 2019). In this study, we show how neurons respond to changes in decoder weights on both timescales before task performance is saturated. Our results regarding stable shared spaces corroborate these past studies but we highlight the importance of the neural variance (and specifically the PV) during learning. While we echo past results that neural activity preferentailly 
324 stays in a constant neural subspace during learning, we found that neurons are also able to

325 quickly increase their private variance when perturbations are introduced. This simultaneous

326 modulation of neural variance whilst maintaining a robust manifold suggests a neural learning

327 strategy that is resilient to perturbations. 


\section{REFERENCES}

Athalye, V.R., Ganguly, K., Costa, R.M., and Carmena, J.M. (2017). Emergence of Coordinated Neural Dynamics Underlies Neuroprosthetic Learning and Skillful Control. Neuron 93, 955970.e5.

Athalye, V.R., Santos, F.J., Carmena, J.M., and Costa, R.M. (2018). Evidence for a neural law of effect. Science (80-. ). 359, 1024-1029.

Athalye, V.R., Carmena, J.M., and Costa, R.M. (2020). Neural reinforcement: re-entering and refining neural dynamics leading to desirable outcomes. Curr. Opin. Neurobiol. 60, 145-154. Carmena, J.M., Lebedev, M.A., Crist, R.E., O’Doherty, J.E., Santucci, D.M., Dimitrov, D.F., Patil, P.G., Henriquez, C.S., and Nicolelis, M.A.L. (2003). Learning to control a brain-machine interface for reaching and grasping by primates. PLoS Biol. 1, 193-208.

Chase, S.M., Kass, R.E., and Schwartz, A.B. (2012). Behavioral and neural correlates of visuomotor adaptation observed through a brain-computer interface in primary motor cortex. J. Neurophysiol. 108, 624-644.

Costa, R.M., Cohen, D., and Nicolelis, M.A.L. (2004). Differential corticostriatal plasticity during fast and slow motor skill learning in mice. Curr. Biol. 14, 1124-1134.

Dangi, S., Orsborn, A.L., Moorman, H.G., and Carmena, J.M. (2013). Design and analysis of closed-loop decoder adaptation algorithms for brain-machine interfaces. Neural Comput. 25, $1693-1731$.

Fee, M.S., and Goldberg, J.H. (2011). A hypothesis for basal ganglia-dependent reinforcement learning in the songbird. Neuroscience 198, 152-170. 
351 Ganguly, K., and Carmena, J.M. (2009). Emergence of a stable cortical map for neuroprosthetic control. PLoS Biol. 7.

353 Ganguly, K., Dimitrov, D.F., Wallis, J.D., and Carmena, J.M. (2011). Reversible large-scale

354 modification of cortical networks during neuroprosthetic control. Nat. Neurosci. 14, 662-669.

355 Georgopoulos, A.P., Kettner, R.E., and Schwartz, A.B. (1988). Primate motor cortex and free 356 arm movements to visual targets in three-dimensional space. II. Coding of the direction of 357 movement by a neuronal population. J. Neurosci. 8, 2928-2937.

358 Gilja, V., Nuyujukian, P., Chestek, C.A., Cunningham, J.P., Yu, B.M., Fan, J.M., Churchland, 359 M.M., Kaufman, M.T., Kao, J.C., Ryu, S.I., et al. (2012). A high-performance neural prosthesis 360 enabled by control algorithm design. Nat. Neurosci.

361 Golub, M.D., Chase, S.M., Batista, A.P., and Yu, B.M. (2016). Brain-computer interfaces for 362 dissecting cognitive processes underlying sensorimotor control. Curr. Opin. Neurobiol. 37, 5336358.

364 Golub, M.D., Sadtler, P.T., Oby, E.R., Quick, K.M., Ryu, S.I., Tyler-Kabara, E.C., Batista, A.P., 365 Chase, S.M., and Yu, B.M. (2018). Learning by neural 366 reassociation/631/378/1595/631/378/2629 article. Nat. Neurosci. 21, 607-616.

367 Izawa, J., Rane, T., Donchin, O., and Shadmehr, R. (2008). Motor adaptation as a process of 368 reoptimization. J. Neurosci. 28, 2883-2891.

369 Jarosiewicz, B., Chase, S.M., Fraser, G.W., Velliste, M., Kass, R.E., and Schwartz, A.B. (2008). 370 Functional network reorganization during learning in a brain-computer interface paradigm. Proc. Natl. Acad. Sci. U. S. A. 105, 19486-19491. 
372 Koralek, A.C., Jin, X., Long, J.D., Costa, R.M., and Carmena, J.M. (2012). Corticostriatal

373 plasticity is necessary for learning intentional neuroprosthetic skills. Nature 483, 331-335.

374 Kording, K.P., Tenenbaum, J.B., and Shadmehr, R. (2007). The dynamics of memory as a

375 consequence of optimal adaptation to a changing body. Nat. Neurosci. 10, 779-786.

376 Moorman, H.G., Gowda, S., and Carmena, J.M. (2017). Control of Redundant Kinematic

377 Degrees of Freedom in a Closed-Loop Brain-Machine Interface. IEEE Trans. Neural Syst.

378 Rehabil. Eng. 25, 750-760.

379 Neuringer, A., Deiss, C., and Olson, G. (2000). Reinforced variability and operant learning. J.

380 Exp. Psychol. Anim. Behav. Process. 26, 98-111.

381 Nowak, D.A., Timmann, D., and Hermsdörfer, J. (2007). Dexterity in cerebellar agenesis.

382 Neuropsychologia 45, 696-703.

383 Oby, E.R., Golub, M.D., Hennig, J.A., Degenhart, A.D., Tyler-Kabara, E.C., Yu, B.M., Chase,

384 S.M., and Batista, A.P. (2019). New neural activity patterns emerge with long-term learning.

385 Proc. Natl. Acad. Sci. 201820296.

386 Ölveczky, B.P., Otchy, T.M., Goldberg, J.H., Aronov, D., and Fee, M.S. (2011). Changes in the

387 neural control of a complex motor sequence during learning. J. Neurophysiol. 106, 386-397.

388 Orsborn, A.L., Dangi, S., Moorman, H.G., and Carmena, J.M. (2012). Closed-loop decoder

389 adaptation on intermediate time-scales facilitates rapid BMI performance improvements

390 independent of decoder initialization conditions. IEEE Trans. Neural Syst. Rehabil. Eng. 20,

$391 \quad 468-477$.

392 Orsborn, A.L., Moorman, H.G., Overduin, S.A., Shanechi, M.M., Dimitrov, D.F., and Carmena, 
J.M. (2014). Closed-loop decoder adaptation shapes neural plasticity for skillful neuroprosthetic control. Neuron 82, 1380-1393.

Sadtler, P.T., Quick, K.M., Golub, M.D., Chase, S.M., Ryu, S.I., Tyler-Kabara, E.C., Yu, B.M., and Batista, A.P. (2014). Neural constraints on learning. Nature 512, 423-426.

Serruya, M.D., Hatsopoulos, N.G., Paninski, L., Fellows, M.R., and Donoghue, J.P. (2002).

401 Shadmehr, R., Smith, M.A., and Krakauer, J.W. (2010). Error correction, sensory prediction, and 402 adaptation in motor control. Annu. Rev. Neurosci. 33, 89-108.

403 Shenoy, K. V., and Carmena, J.M. (2014). Combining decoder design and neural adaptation in 404 brain-machine interfaces. Neuron 84, 665-680.

405 Taylor, D.M. (2002). Direct Cortical Control of 3D Neuroprosthetic Devices. Science (80-. ). $406 \quad 296,1829-1832$.

407 Wander, J.D., Blakely, T., Miller, K.J., Weaver, K.E., Johnson, L.A., Olson, J.D., Fetz, E.E., 408 Rao, R.P.N., and Ojemann, J.G. (2013). Distributed cortical adaptation during learning of a 409 brain-computer interface task. Proc. Natl. Acad. Sci. U. S. A. 110, 10818-10823.

410 Wei, K., and Körding, K. (2009). Relevance of error: What drives motor adaptation? J.

411 Neurophysiol. 101, 655-664.

412 Wessberg, J., and Nicolelis, M.A.L. (2004). Optimizing a linear algorithm for real-time robotic 413 control using chronic cortical ensemble recordings in monkeys. J. Cogn. Neurosci. 16, 1022- 
bioRxiv preprint doi: https://doi.org/10.1101/817346; this version posted March 4, 2020. The copyright holder for this preprint (which was not certified by peer review) is the author/funder, who has granted bioRxiv a license to display the preprint in perpetuity. It is made available under aCC-BY-NC-ND 4.0 International license.

4141035.

415 


\section{METHODS}

\section{Surgical Procedures and Electrophysiology}

418 All procedures for each of the three experiments were conducted in compliance with the NIH

419 Guide for the Care and Use of Laboratory Animals and were approved by the University of

420 California, Berkeley Institutional Animal Care and Use Committee.

421 Experiment 1

422 One adult male rhesus macaque was chronically implanted with 64-channel Teflon-coated

423 tungsten microelectrode arrays. Implants were in the arm regions of primary motor cortex (M1)

424 and dorsal premotor cortex (PMd) in the left hemisphere. There was an additional implant in the

425 arm area of M1 in the right hemisphere, totaling three implants. Unit activity was recorded using

426 a MAP system (Plexon Inc., Dallas, TX) and sorted with an online spike-sorting application

427 (Sort Client; Plexon). Units stable across days were used for decoding (Ganguly and Carmena, 428 2009).

429

431 Two adult male rhesus macaques were chronically implanted with 128-channel microwire

432 electrode arrays. Implants were bilateral, targeting the arm areas of M1. Neural activity was

433 recorded using the same MAP system as in Experiment 1. Multi-unit activity was sorted using

434 Sort Client for Monkey S and channel-level activity was used for Monkey J (Orsborn et al., 435 2014). 
438 Two adult male rhesus macaques were chronically implanted with microwire electrode arrays

439 with 128 channels in each hemisphere, targeting arm areas of M1 and PMd in Monkey G and

440 primary sensory cortex (S1) and M1 in Monkey C. Neural activity was filtered and recorded

441 using an OmniPlex system (Plexon Inc., Dallas, TX). Single and multi-unit activity was sorted

442 with a template-matching algorithm (PlexControl) (Moorman et al., 2017).

\section{BMI Task}

445 In all three of the experiments we analyzed, monkeys performed a center-out BMI task. Subjects

446 were instructed to enter and hold in the center target to initiate a trial. Upon hold completion, one

447 of eight peripheral targets would appear. Subjects then moved the cursor towards the target after

448 this go cue. Trials were successful if the cursor entered and stayed in the peripheral target for a

449 short hold time $(\sim 250 \mathrm{~ms})$ for each experiment. Decoders were trained differently in the three

450 experiments (outlined below), but in all cases, subjects were familiar with the task structure

451 before attempting control with BMI. Therefore, subjects' performance improvement can be

452 attributed to BMI learning rather than task learning. Once decoder weights were held fixed each

453 day, task control was achieved by modulating neural activity, which was recorded and fed

454 through the decoder to produce cursor movements. In order to observe neural correlates of

455 coordinated control, we analyzed movement portions (i.e. leaving center target to entering

456 peripheral target) of successful trials for each experiment (red box, Figure 1B). 
459 In Experiment 1, two macaques were instructed to complete a BMI center-out task as described

460 above. After achieving proficient control with an "old" decoder, a new biomimetic decoder was

461 introduced. This decoder was trained on Day 1 (Figure 1 C). While many weights were similar

462 between the two decoders, many of the weights were significantly different (Ganguly and

463 Carmena, 2009). Each day, both decoders were used for BMI control in a series of two blocks.

464 To track proficiency with each decoder, the percent of correct trials was calculated for each

465 block for each day. For each decoder, a linear filter (Wiener filter) was used to fit neural activity

466 to kinematic activity in the elbow and shoulder joints during a manual reaching task using a

467 BKIN KINARM exoskeleton robot. Decoder outputs were then translated into Cartesian space

468 by using a Jacobian matrix. For more details, we redirect the reader to Ganguly and Carmena, 4692009.

470

$471 \quad$ Wiener Filter

472 The Wiener filter model assumes that the cursor velocity is a linear combination of neural

473 activity across small differences in time. In other words, the output of the decoder at time $t$ is

474 determined by a weighted sum of the neural activity from $t-k$ for some set of $k$ representing

475 the time lags. More specifically:

476

477

$$
y(t)=\boldsymbol{b}+\sum_{k=0}^{M} \boldsymbol{a}(k) * x(t-k)+\epsilon(t)
$$


479 where $\epsilon$ refers to any residual errors and $M$ represents the number of time lags used (in

480 Experiment 1, this was 10). The parameters $\boldsymbol{a}$ and $\boldsymbol{b}$ were determined to optimally fit the model

481 (Ganguly and Carmena, 2009; Wessberg and Nicolelis, 2004).

482

\section{Experiment 2}

484 The second experiment we analyzed was originally conducted by Orsborn et al in 2014 . To

485 summarize, prior to BMI control, both subjects were trained to conduct the manual control

486 version of the center-out task using the KINARM. An initial Kalman filter (KF, outlined below)

487 decoder was fit on day 1 on these manual reaches. Closed-loop decoder adaptation (CLDA) was

488 then used to better fit decoder weights to the neural intentions (Orsborn et al., 2012). To briefly

489 summarize, CLDA updates decoder weights by inferring intentions of the subject based on task

490 goals. Decoder weights were updated through a weighted average of old and new decoder

491 weights. CLDA was run at the beginning of day 1 to achieve some level of control ( $20 \%$

492 accuracy). Over days, decoder weights were occasionally refit with CLDA, sometimes in

493 conjunction with neuron swaps to recover performance. After 12 days, the procedure was

494 repeated for Monkey J with initial performance elevated to $40 \%$ accuracy. For further details

495 regarding CLDA, we redirect readers to the methods outlined in Orsborn et al., 2012.

497 Experiment 3

498 In Experiment 3, two monkeys (Monkeys $\mathrm{C}$ and $\mathrm{G}$ ) controlled a four degree-of-freedom (DOF)

499 kinematic chain in a center-out task. Subjects were first trained to perform a cursor center-out

500 task using manual control with a KINARM exoskeleton as in previous studies, then were

501 transitioned to BMI control of a 2D cursor before learning to perform the full 4 DOF task with 


$$
\mathbf{A}=\left[\begin{array}{ccc}
\mathbf{I} & \Delta t \mathbf{I} & 0 \\
\mathbf{0} & 0.8 \mathbf{I} & 0 \\
0 & 0 & 1
\end{array}\right], \mathbf{W}=\left[\begin{array}{ccc}
\mathbf{0} & \mathbf{0} & 0 \\
\mathbf{0} & 0.1 \mathbf{I} & 0 \\
0 & 0 & 0
\end{array}\right]
$$
where $\mathbf{I}, \mathbf{0}$ are identity and 0 matrices, respectively, and $\Delta t$ is $100 \mathrm{~ms}$. Note that in these models,

523 A contains a scaling factor (0.8) in order to decay the velocities over time.

BMI control. Movements of the kinematic chain were constrained to two dimensions thereby creating a kinematically redundant system. A KF decoder fit neural activity to four individual joint angle velocities. Visual feedback of the kinematic chain moving from target to target was used to seed the KF decoder and CLDA was used to update neural intentions each day. Unlike Experiment 2, CLDA was run until the subjects’ performance saturated each day $(\sim 85 \%$ accuracy). Since the percent correct was saturated and stable across days, task improvement was tracked by decreasing reach times. We refer readers to Moorman et al., 2017 for more experimental details.

\section{Kalman Filter}

Here, we briefly describe the Kalman filter model for BMI decoding used in Experiments 2 and 3. For more details, we refer the reader to Orsborn et al., 2014 and Moorman et al., 2017. In general, the state-space (e.g. kinematic space) is represented by $x_{t}=\left[p_{t} v_{t} 1\right]$ where $p_{t}$ represents the positions of the effector and $v_{t}$ represents the velocities of the effector. In Experiment 2, the state-space contains the horizontal and vertical positions and velocities. In Experiment 3, the state-space refers to the joint angles and angular velocities of the 4 DOF kinematic chain. The Kalman Filter model assumes that $x_{t}$ varies as $x_{t+1}=\mathbf{A} x_{t}+w_{t}$ where $w_{t}$ is a noise term $\left(w_{t} \sim N(0, \mathbf{W})\right)$. $A$ advances the state using the current positions and velocities and $W$ is set up so as to allow the joint velocities to evolve independently: 
524 The observations $y_{t}$ represent the neural activity in the past $100 \mathrm{~ms}$. The KF model of neural

525 firing assumes that $y_{t}=\mathbf{C} x_{t}+q_{t}, q_{t} \sim N(0, \boldsymbol{Q})$. The KF estimates $x_{t}$, the state variable, from

526 the previous $t$ observations $\left\{y_{0}, \ldots, y_{t}\right\}$ and produces the state estimate $x_{t}$ and confidence $\mathbf{P}_{t}$. The

527 KF uses the last estimate $x_{t-1}$ and advances that belief to produce $x_{t \mid t-1}=A x_{t-1}$, and then

528 updates the prediction when a new observation becomes available: $x_{t}=x_{t \mid t-1}+K_{t}\left(y_{t}-\right.$

$\left.529 C x_{t \mid t-1}\right)$. The Kalman gain $\mathbf{K}_{t}$ is then determined by the model parameters $\{\mathbf{A}, \mathbf{C}, \mathbf{W}, \mathbf{Q}\}$ and $\mathbf{P}_{t}$.

530 Since our model parameters are fixed, $\mathbf{K}_{t}$ converges to a steady-state $\mathbf{K}$, and we estimate the

531 kinematics to follow the simpler equation:

$$
x_{t}=(\mathbf{I}-\mathbf{K C}) \mathbf{A} x_{t-1}+\mathbf{K} y_{t}
$$

\section{Factor Analysis}

535 Factor analysis (FA) was used in this study to approximate low-dimensional manifolds; here we provide an outline of the method (Athalye et al., 2017). FA decomposes the neural activity into

537 three components: the mean firing rates $(\mu)$ of each neuron, the population-level coordinated

538 firing activity $\left(x^{\text {shared }}\right)$, and the remaining component corresponding to each neuron's

539 uncorrelated activity to the rest of the population $\left(x^{\text {private }}\right)$. That is,

$$
x=\mu+x^{\text {private }}+x^{\text {shared }}
$$

541 These latter two components have covariance matrices $\Sigma^{\text {private }}$ and $\Sigma^{\text {shared }}$ with dimensions

$542 N \times N$, where $N$ is the number of neurons. The combination of these two parts yields the total 543 variance,

$$
\Sigma^{\text {total }}=\Sigma^{\text {shared }}+\Sigma^{\text {private }}
$$


545 The dimensionality was estimated by using cross-validated log-likelihood to determine the number of factors that would best describe held-out data such that $\sim 90 \%$ of the shared variance

547 could be captured (Athalye et al., 2017).

Importantly, only units stable across all days for each experiment were used for these analyses.

Since we were interested in how the same ensemble of units adapted over learning, including all units used for each day would contaminate across-day results.

\section{Private Variance}

554 We define private variance $(\mathrm{PV})$ as an $N \times N$ covariance matrix where the diagonals represent 555 independent variances for each of the $N$ neurons. In Experiment 1, the PV was calculated for 556 each target and each block. Average PV values were found by averaging across the 8 targets for

557 each block. Similarly, PV was calculated for each day (and one day after) a decoder was refit in

558 Experiment 2. This gave us PV values before and after refitting and a t-test was used to

559 determine differences in PV. Furthermore, to track how PV changed over days with stable

560 decoders, PV was calculated for days without decoder changes as well. In Experiment 3, since

561 CLDA was run each day of the experiment and percent accuracy on Day 1 was already as good 562 as the last day (i.e. proficient control), we instead examined the correlation of the decoder for 563 each day with the decoder on the first day as a metric for the difference between decoders. We 564 then plotted this correlation against the average private variance over all neurons for that day 565 (Figure 3E). Note that this correlation was shown for stable units in Figure 3E; Supplemental 566 Figure 3A shows this correlation calculated using stable channels for Monkey G, which include 
567 channels that were used in the BMI task but were either physiologically different across recording days or contained no physiological signal.

\section{Shared / Total Variance}

571 We defined the shared/total variance ratio (SOT) as

$$
\text { SOT }=\frac{\operatorname{trace}\left(\Sigma^{\text {shared }}\right)}{\operatorname{trace}\left(\Sigma^{\text {total }}\right)} \text {. }
$$

Intuitively, this allowed us to measure the proportion of neural variance that was coordinated. In

574 order to track how neurons in the population changed coordination patterns over learning, we

575 only considered units that were stable across the entirety of the experiments for these analyses,

576 determined by waveform and inter-spike interval statistics. In all experiments, the SOT was

577 calculated for each block/day for all stable units. In Experiment 3, Monkey G had several

578 "unstable" units throughout the experiment which were not considered in these analyses. In

579 addition, a variety of noisy channels were included as decoder inputs. Figures 3 and 4 show

580 results with stable units for Monkey G, however the results still hold using all stable channels

581 (Supplemental Figures 4-5).

\section{Shared Space Alignment}

584 In order to determine how similar manifolds were between experimental blocks (or days), we calculated the "alignment" between shared spaces (Athalye et al., 2017). Geometrically, it shows

586 how much of one subspace projects onto a second as a fraction ranging from 0 to 1 . Moreover, a

587 perfectly aligned subspace results in an alignment value of 1 while orthogonal subspaces have an

588 alignment of 0 . To calculate the shared space alignment, we find the projection of the shared

589 variance of block (or day) $A$ onto the shared space of block (or day) $B$. 


$$
\text { shared alignment }=\frac{\operatorname{trace}\left(\mathrm{P}_{\left.\mathrm{U}^{\mathrm{B}} \Sigma^{\mathrm{A}, \text { shared }} \mathrm{P}_{\mathrm{U}^{\mathrm{B}}}^{\mathrm{T}}\right)}\right.}{\operatorname{trace}\left(\Sigma^{\mathrm{A}, \text { shared }}\right)}
$$

591 where $P_{U}^{B} \in R^{N \times N}$ is an projection matrix onto the shared subspace of block $B\left(\operatorname{col}\left(U^{B}\right)\right)$, and $592 \Sigma^{A, \text { shared }}$ is the shared variance of block $A$.

594 To measure how similar shared spaces were between decoder changes, we first found the shared 595 alignment between pairwise days. In Experiment 1, comparisons were then made between all 596 decoder block changes and all other pairwise comparisons. Similarly, to test if shared spaces

597 were aligned on short and longer timescales in Experiments 2 and 3, shared alignments between 598 adjacent days were compared against all other pairwise combinations of days. For each experiment, a Kolmogorov-Smirnov test was used to determine if the distributions of shared

600 alignment values were significantly different.

601

\section{Preferred Direction and Modulation Depth}

603 We also looked at the correlation of modulation depth and private variance. Cursor kinematics 604 were separated into eight 45-degree bins and corresponding firing rates were used to determine 605 the preferred tuning direction (PD) for each neuron by fitting the firing rate $f$ to a cosine function with mean firing rate $\mu$ :

$$
f=\left[\begin{array}{lll}
B_{1} & B_{2} & B_{3}
\end{array}\right] \times\left[\begin{array}{c}
\mu \\
\sin (\theta) \\
\cos (\theta)
\end{array}\right]
$$
regression. PD was calculated as $\mathrm{PD}=\arctan \left(B_{2} / B_{3}\right)$, resolved to the correct quadrant. Modulation depth (MD) was then defined as the peak-to-peak amplitude of this curve.

611 Intuitively, it shows how specific neurons fire in their PD and is calculated as 


$$
\mathrm{MD}=\sqrt{B_{1}^{2}+B_{2}^{2}}
$$

613 To track how MD and PV changed together over learning, we correlated the changes in MD (i.e.

614 derivative) with the changes in PV across days. As before, Figure 4A (inset) shows the results

615 when considering only stable units for Monkey $\mathrm{G}$ to remain consistent with the other animals.

616 However, the results are consistent if all stable channels are used as well (Supplemental Figure

$6173 \mathrm{~B})$.

\section{Statistical Analyses and Testing}

620 We used t-tests to reject null hypotheses with a confidence of 5\% and used linear regressions and

621 correlations to fit trends. Kolmogorov-Smirnov tests were used to test whether distributions were

622 the same. Wilcoxon rank-sum tests were used to compare private variance distribution

623 differences. Where reported, error bars represent standard error of the mean over 8 targets. All

624 analyses were done in MATLAB by writing custom scripts.

625 


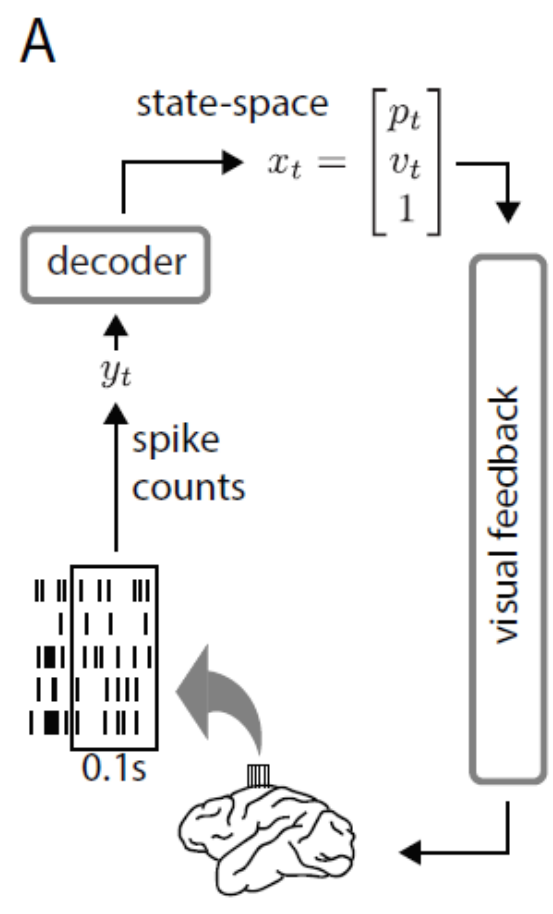

C

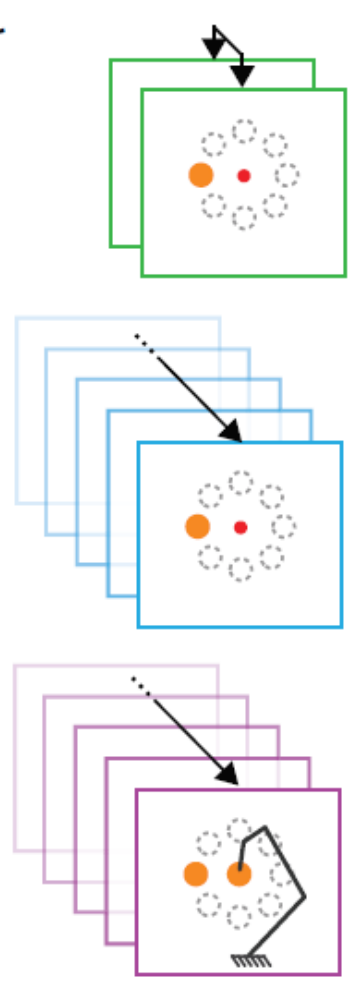

B

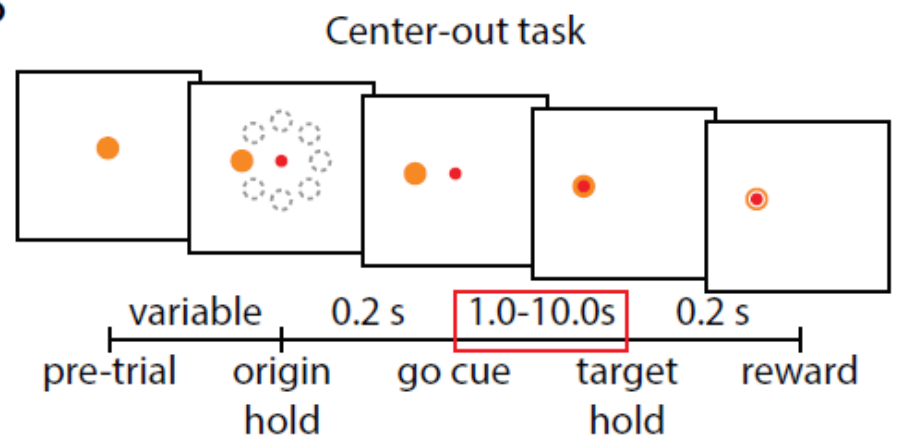

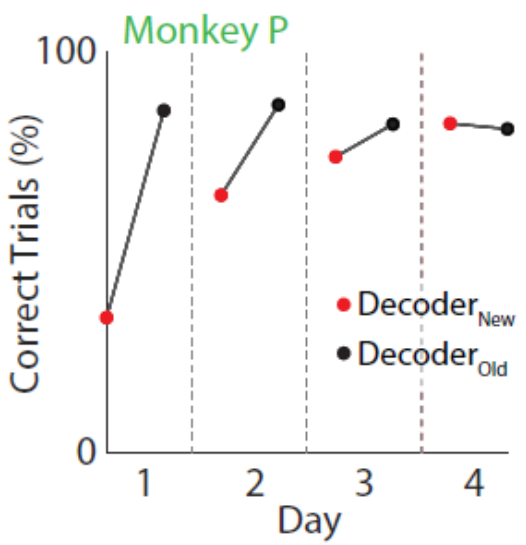
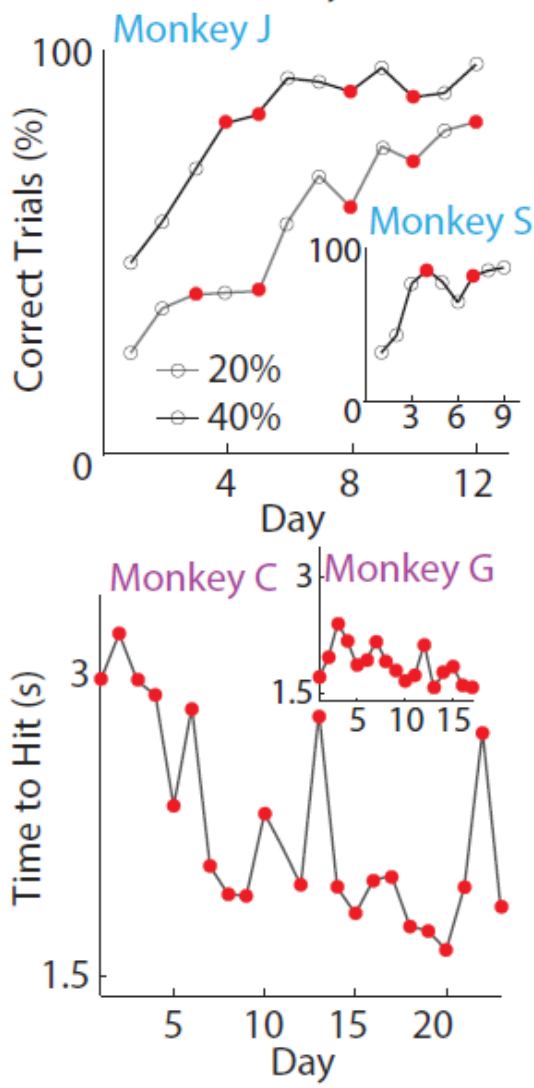

A. Closed-loop BMI. Neural signals were recorded from motor cortices in the brain of rhesus macaques. Spikes were recorded and decoded to update a state-space vector, moving the effector in the animals' visual field (adapted from Moorman and Gowda et al., 2017). 
B. In all three experiments, monkeys were instructed to perform a center-out task, moving the effector from a center target to one of eight randomly selected peripheral targets. Successful trials were rewarded with juice. Only rewarded trials during movement periods (red box) were analyzed in this study (adapted from Moorman and Gowda et al., 2017).

C. Three experiments were analyzed in this study. Green, two decoders were used each day (Ganguly and Carmena, 2009). After proficient control of one decoder (black), a new decoder was introduced at the beginning of each successive day (red). After a period each day, decoders were swapped back to the previously learned decoder. Percent accuracies were calculated as the ratio of successful trials to number of self-initiated trials.

Blue, decoders were re-adapted periodically across training and neural populations occasionally changed from day-to-day (Orsborn et al., 2014). Monkeys were trained to perform a center-out task with decoders of varying amounts of adaptation (CLDA) as defined by the initial performance of each time series (i.e. $20 \%$ and $40 \%$ ). Monkey $\mathbf{J}$ was trained on two time series. After 12 days of $20 \%$ CLDA, he was switched to a $40 \%$ decoder. Monkey S remained on a $20 \%$ decoder for the entirety of the experiment. Red dots indicate decoder re-adaptation.

Purple, a virtual arm with 4 degrees of freedom (DOF) was controlled with a new decoder each day (Moorman and Gowda et al., 2016). Monkeys trained to move the effector in 2D space and improved reach times over learning (Monkey $C, R^{2}=0.431, p=$ 6.6e-4; Monkey $\left.\mathrm{G}, \mathrm{R}^{2}=0.298, \mathrm{p}=0.0233\right)$. CLDA was performed each day and percent accuracy was saturated from Day 1 for both animals ( $~ 80 \%$ correct trials across all days). 


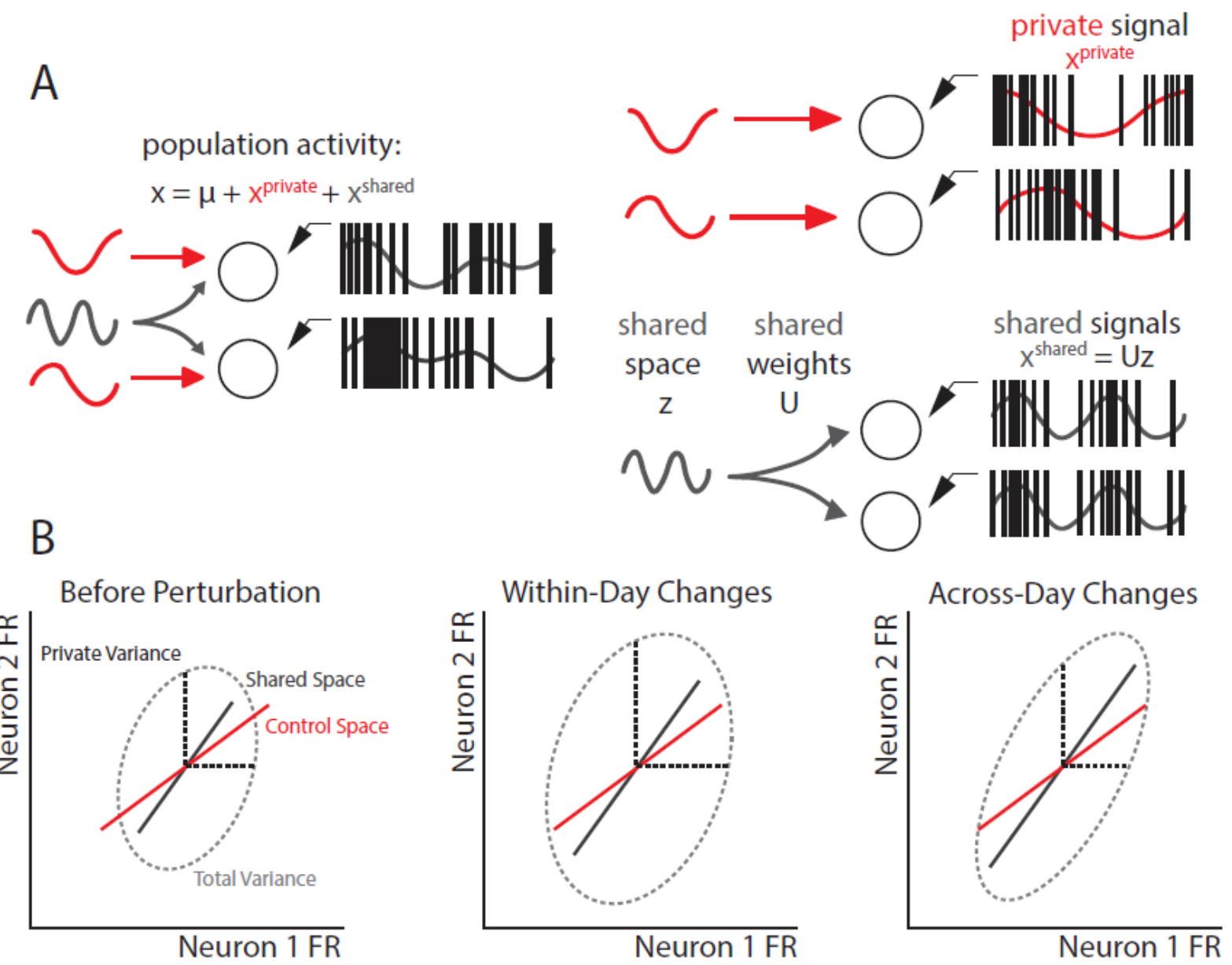

Figure 2: Factor analysis model predictions

A. Factor Analysis (FA) model. Neural signals can be demeaned $(\mu)$ and decomposed into covarying signals (shared) and uncorrelated signals (private). Intuitively, shared signals indicate a low-dimensional neural space where neurons fire in coordinated ways (Athalye et al., 2017).

B. Predictions of neural subspace changes in response to decoder re-adaptation. Neural firing patterns between two neurons are shown in the dashed ellipses. Private variance (black, dashed) explains the variance that is independent from other neurons. Before decoder re-adaptation, there is an intrinsic shared space (gray). Since CLDA fits to neural 
intentions, we expect the decoder plane (control space, red) to be fairly aligned with the

666

neural subspace (shared space, gray). Lengths of lines indicate amount of variance 
A

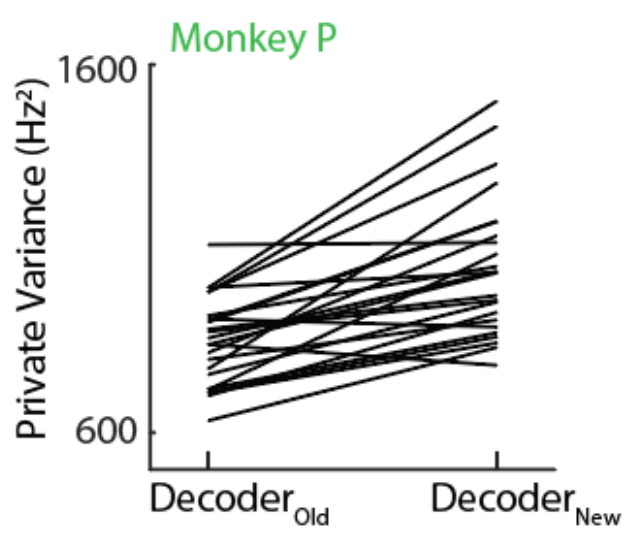

C

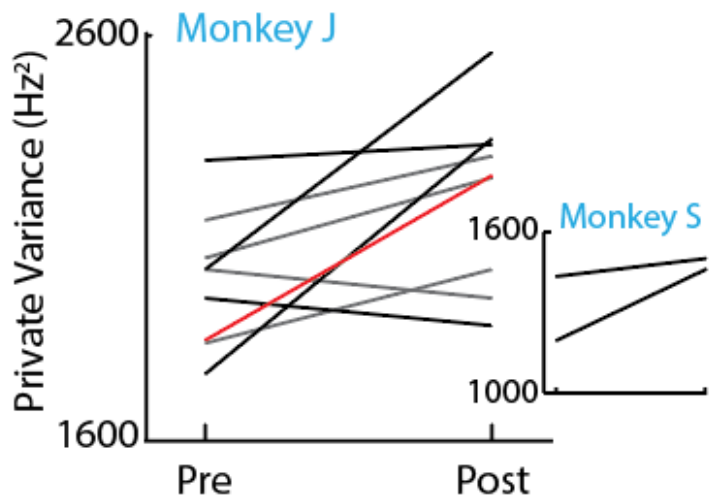

$\mathrm{E}$

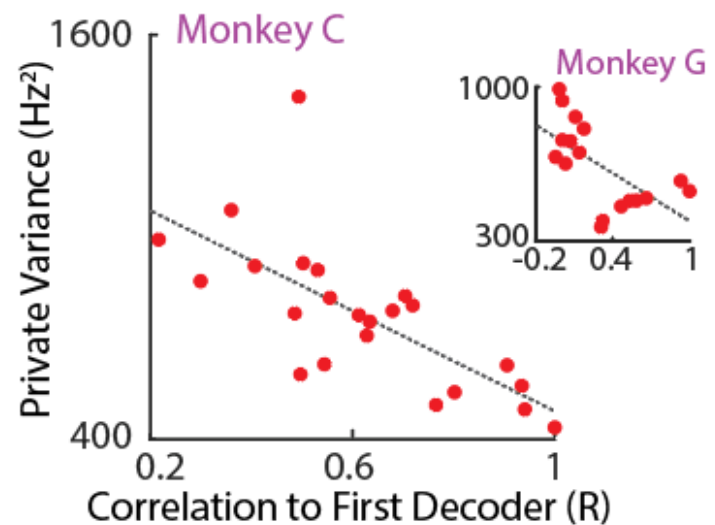

B

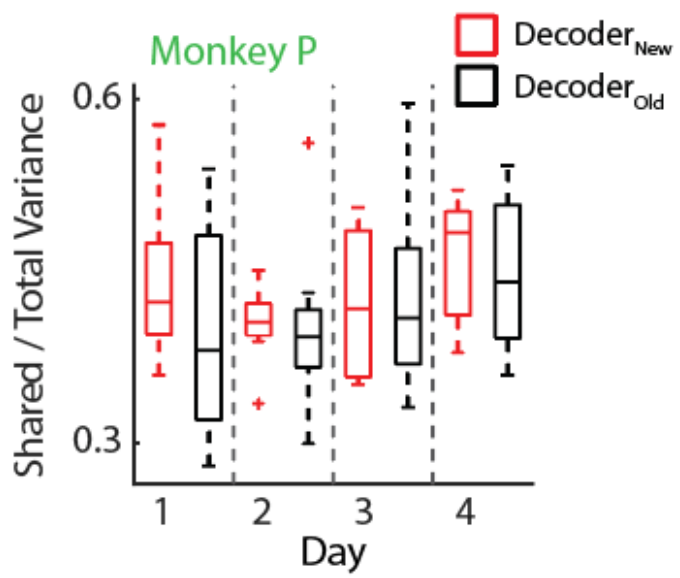

D

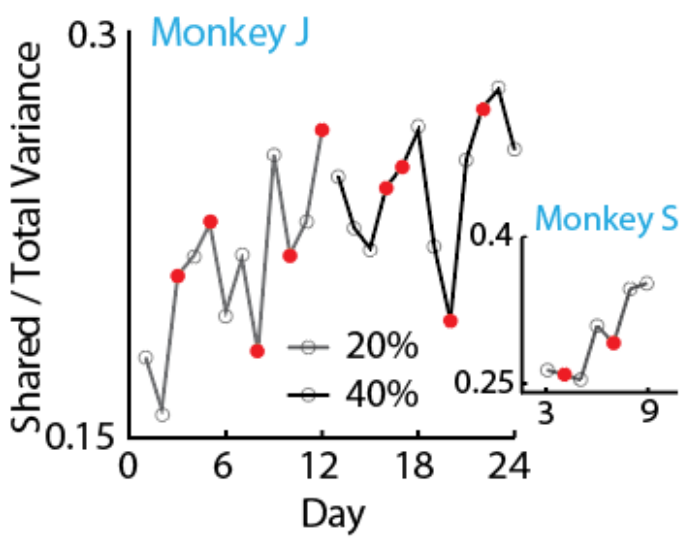

$\mathrm{F}$

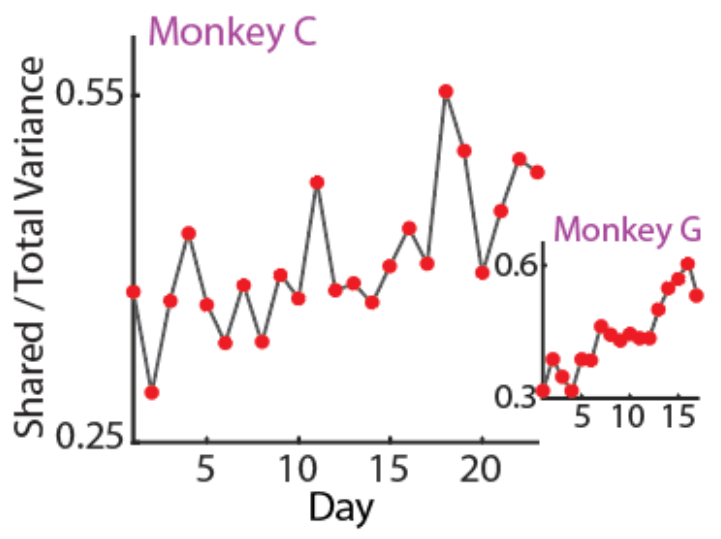

Figure 3: Exploratory and consolidated patterns.

A. Private variance (PV) changed within day. Using the new decoder increased the PV at the beginning of each day (t-test; $\mathrm{p}=1.5 \mathrm{e}-6)$. 
B. Consolidated patterns did not change within day. The shared-to-total variance ratio (SOT)

682 was stable each day and did not change as a result of switching decoders.

C. PV increases when decoders are re-adapted. Across both $20 \%$ and $40 \%$ time series, PV increased when decoders were refit with CLDA. The red line indicates the transition from the $20 \%$ experiment to the $40 \%$ experiment. (Wilcoxon rank-sum test; Monkey J, p = 0.0315; Monkey S n.s., only two decoder re-adaptations were performed). $\mathrm{p}=2.8 \mathrm{e}-4$; Monkey $\mathrm{S}, \mathrm{R}=0.81, \mathrm{p}=6.1 \mathrm{e}-3)$.

E. PV was higher in cases where decoder weights were more different from the first day. (correlation; Monkey C, R = -0.70, p = 2e-4; Monkey G, R = -0.63, p = 6.3e-3). 
A

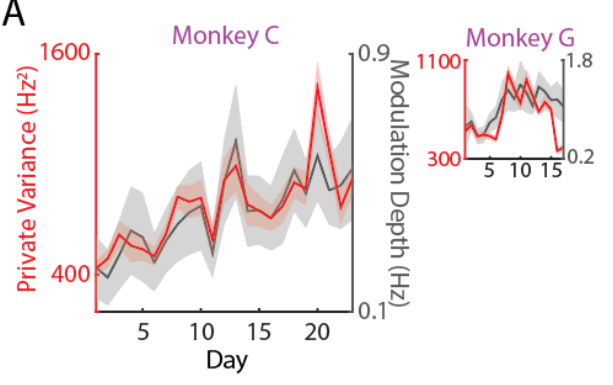

B

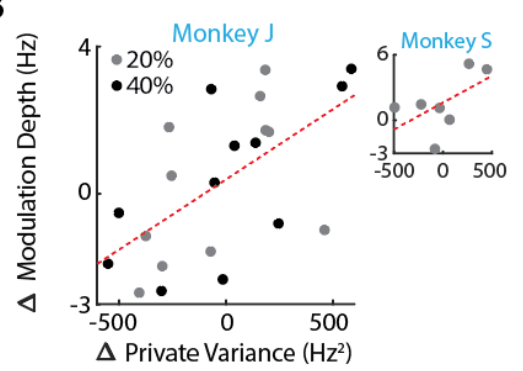

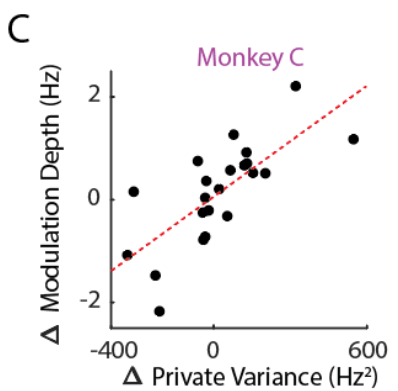

695

696

697

698

699

700

701

702

703

704

705

706

707

\section{Figure 4: Modulation depth may facilitate PV changes.}

A. PV fluctuations are proportional to changes in MD (correlation; Monkey $\mathrm{C}, \mathrm{R}=0.86, \mathrm{p}<$ 1e-5; Monkey G, R = 0.63, p = 7e-3). Lines indicate mean values of PV and MD; shaded regions show s.e.m. across targets for each day. While the changes in PV and MD were not significantly correlated for Monkey $\mathrm{G}$ in (C), the overall trends are proportional.

B. Changes in PV were closely linked with changes in modulation depth (MD). Each dot represents one decoder change. Regression was conducted on combined data across the 24 days for Monkey $\mathrm{J}$ (correlation; $\mathrm{R}=0.63, \mathrm{p}=0.0013$ ). Correlation was not significant for Monkey $\mathrm{S}$ due to too few data but still shows same trends (correlation, $\mathrm{R}=0.569, \mathrm{p}=$ $0.18)$.

C. Same as in (B) for Monkey $\mathrm{C}$ in Experiment 3 (correlation; $\mathrm{R}=0.73, \mathrm{p}=1 \mathrm{e}-4$ ). Results were not significant for Monkey $\mathrm{G}$ due to large fluctuations in channels used for recording from day-to-day. 